\title{
El fin ético no naturalista de la neuroeducación*
}

\author{
The non-naturalistic ethical end of neuroeducation
}

\author{
JAVIER GRACIA CALANDIN
}

Departamento de Filosofía del derecho, moral y política. Universitat de ValÈnCia

Artículo recibido: 23 de junio de 2017

Solicitud de revisión: 13 de noviembre 2017

Artículo aceptado: 9 de enero de 2018

\section{Resumen}

El objetivo de este artículo es aclarar cuál es el fin último de la neurociencia educativa. Para ello nos adentramos en el fecundo y a veces controvertido diálogo entre neurociencia y educación que especialmente en la última década ha cobrado mayor importancia. La hipótesis que presentamos es que la introducción de la perspectiva ética contribuye positivamente a esclarecer el fin propio de la neuroeducación. A la luz de una neuroética no naturalista la educación puede abrirse a un nuevo enfoque evolutivo que no reduzca el fin de la educación al simple valor adaptativo.

Palabras clave: Neurociencia, educación, ética, neuroeducación y neuroética.

\begin{abstract}
This paper aims to clarify the main goal of neuroeducation. To do this, I focus on the fruitful but often controversial discussion between neuroscience and education, which has been especially relevant in the last decade. The hypothesis I present is that the introduction of the ethical perspective positively affects the clarification of neuroeducation's proper end. In the light of the non-naturalistic approach to neuroethics that I hold, education could be opened up to a new evolutionary approach that does not reduce the end of education to mere adaptive value.
\end{abstract}

Keywords: Ethics, Education, Neuroscience, Neuroethics and Neuroeducation.

* Esta contribución se enmarca en el Proyecto de Investigación Científica y Desarrollo Tecnológico "Neuroeducación moral, democracia deliberativa y políticas de desarrollo humano sostenible" FFI2016-76753-C2-1-P. 


\section{EL PODER SEDUCTOR DE LA NEUROCIENCIA EN LA EDUCACIÓN}

En una interesante investigación realizada para analizar el diálogo entre educación y neurociencia, Paul Howard-Jones se hace eco de la opinión de algunos educadores de que el poder seductor de la ciencia podría dejar a la educación a merced de los neuromitos. Tanto educadores como Guy Claxton como neurocientíficos como Mark Johnson y Daniel Wolpert alertaban contra la tendencia de pensar entusiastamente en la neurociencia como la panacea para resolver los problemas que surgen en el aula.

La educación ha sido una disciplina insegura, que siempre ha tenido que preguntarse a sí misma, ¿đónde reside nuestra garantía? ¿Cómo justificamos y racionalizamos lo que hacemos? Y ahora, particularmente cuando los medios y los fines de la educación son acaloradamente rebatidos. Esta inseguridad puede hacernos de algún modo crédulos como profesión y vulnerables a las pretensiones de una disciplina que irrumpe y dice "yo soy el adecuado científico del conocimiento. Me baso en puros datos y puedo decir qué hacer» (Howard-Jones, 2010: 65).

En una sociedad en la que la ciencia y la tecnología gozan de tanto prestigio, tampoco la educación escapa al poder seductor de las promesas del discurso cientificista. Precisamente, la educación con más motivo que otras disciplinas es propensa a ser colonizada por las pretensiones cientificistas puesto que constituye uno de los pilares y bienes básicos de la sociedad. Pero tal vez por ello y lejos de rechazar de plano cualquier aporte de la ciencia sea más necesario si cabe hacerse cargo de los límites y riesgos de la pretensión cientificista de poder servir como solución a todos los problemas que trae consigo la educación.

El objetivo de este artículo es plantear el interrogante acerca de cuál ha de ser la contribución de la neurociencia a la educación. No para alimentar una actitud de temor replegada sobre sí misma, sino para estar alerta, en actitud filosófica de desvelamiento, y así alcanzar una mejor comprensión de la función que le corresponde a la neurociencia en relación con la educación. ${ }^{1}$

En primer lugar, se plantea cómo ha de entenderse la relación entre neurociencia y educación y si es la neurociencia la que debe guiar la práctica educativa. En segundo lugar, se introduce la hipótesis de si la ética

1 Como es bien sabido, al menos desde finales de los años noventa del siglo pasado a raíz del artículo de Bruer (1997), se ha venido cuestionando cómo entender la relación entre neurociencia y educación y si acaso es posible establecer un puente entre ambas disciplinas. 
como tercer elemento de la relación entre neurociencia y educación puede ayudar a esclarecer el fin propio de la neuroeducación. En tercer y último lugar, se argumenta a favor del fin ético de la neuroeducación y en contra de la visión naturalista que reduce la neuroeducación a factores adaptativos.

\section{2. ¿CÓMO ENTENDER LA RELACIÓN ENTRE NEUROCIENCIA Y EDUCACIÓN?}

Para no incurrir en una falsa atribución de la neurociencia como panacea para resolver los problemas de la educación, Paul Howard-Jones aboga por diferenciar los diferentes tipos de «evidencia» de una y otra. En el caso de la neurociencia solo se trata de un elemento que permite explicar cómo funcionan determinados procesos de enseñanza y aprendizaje: «la enseñanza sirve para validar un elemento bastante particular de comprensión que ocupa un lugar muy definido dentro de un cuerpo de conocimiento progresivamente construido» (Howard-Jones, 2010: 67). Pero, a su juicio, es la educación la que permite introducir «valores e ideas sobre una escala mucho mayor» porque se plantea "cuestiones de valor».

A continuación, sin embargo, añade que ambos objetivos, el de la neurociencia y el de la educación están directamente relacionados y no es adecuado confinar la neurociencia a laboratorios ni vetar la entrada de los educadores a las explicaciones neurocientíficas para que de este modo puedan llegar a comprender los procesos neuronales del aprendizaje. Una muestra de lo primero sería la «neurociencia cognitiva social y educacional», y de lo segundo, educadores con conocimientos en neurociencia. A juicio de Howard-Jones es necesaria esta labor de mutua colaboración entre educadores y neurocientíficos porque la neurociencia no puede proporcionar respuestas completas sino más bien explicar cómo funciona el cerebro.

Otros autores han insistido en la importancia de tender puentes entre neurociencia y educación, si bien han considerado las dificultades que esto plantea.A juicio de Goswami y Szücks (2011) es necesario entender adecuadamente los mecanismos subyacentes de nuestro cerebro con vistas a anticipar posibles disfunciones. Centrados en los problemas de la dislexia y la discalculia respectivamente, Goswami y Szücks señalan que el paso básico consiste en comprender los mecanismos del sistema motor en todo el cerebro guiándose por cuatro principios. Añaden que esta investigación no será 
útil para educadores a corto plazo, sin embargo, no hay que perder de vista la implicación que para la educación han de tener los descubrimientos neurocientíficos y «la significación real de los datos extraídos de neuroimágenes consistirá en comprender cómo estas diferencias o cambios se relacionan con la práctica educativa» (Goswami \& Szücks, 2011: 654).

Otros autores como J. S. Bowser (2016), sin embargo, han sido mucho más reticentes a estrechar el vínculo entre neurociencia y educación, y cuestionan que las investigaciones neurocientíficas puedan realmente suponer una mejora en la práctica educativa.A su juicio, no hay muestras de que los conocimientos adquiridos en el campo de la neurociencia hayan generado nuevos métodos didácticos y tampoco que en el futuro se pueda llegar a crear estrategias de una enseñanza más efectiva. Para Bowser, la pretensión de que la neurociencia sirva como guía y solución a los problemas del aula es generadora de neuromitos que hacen un flaco favor a la práctica educativa.

La respuesta al reciente artículo de Bowser (2016) no se hizo esperar y un grupo de reconocidos investigadores en neuroeducación tales como Paul A. Howard-Jones, Daniel Ansari, Bert De Smedt, Diana Laurillard, Sashank Varma, Brian Butterworth, Usha Goswami y Michael S. C. Thomas (2016) han rebatido su tesis y han señalado cuál debe ser esta relación y diálogo entre la neurociencia y la educación en lo que ha dado en llamarse «neurociencia educativa» o «neuroeducación».A juicio de estos autores, las críticas de Bowser contra la neurociencia educativa se basan en una mala forma de entender la relación entre la neurociencia y la educación y lo que ellos proponen es llevar a cabo una «ruta intermedia y compleja». No se trata de trazar un plan que vaya desde el escaneado cerebral a las clases, antes bien:

La neurociencia puede decir dónde mirar - esto es, qué funciones neuronales están sanas o dañadas y de qué modo operar- pero este conocimiento debe ser transformado por principios pedagógicos en intervenciones. Dichas intervenciones pueden ser evaluadas tanto en base a su efectividad en las pruebas de comportamiento en el contexto educativo, como por los marcadores neuronales en los estudios de laboratorio, como medios para examinar los mecanismos por los cuales han ocurrido algunos efectos (Howard-Jones et al., 2016: 622).

No voy a detenerme a explicar los seis aspectos que Howard-Jones y sus colaboradores (2016) proponen para llevar a cabo su «ruta intermedia y compleja» de neurociencia educativa. No obstante, sí que me parece que es importante destacar el énfasis que todos estos autores ponen en la importancia de trazar bien estos puentes entre neurociencia y educación 
para no generar o alimentar neuromitos en el ámbito educativo (Dekker $e t$ al., 2012; Howard-Jones, 2010).

Por mi parte, me sumo a los intentos por esclarecer la relación entre educación y neurociencia y me voy a detener en el interrogante acerca del fin de la neuroeducación. Comenzábamos este artículo haciéndonos eco de una preocupación que algunos educadores tienen acerca del poder seductor de la neurociencia dado el protagonismo creciente que esta está cobrando en el modo de entender la forma de vida de las personas. Efectivamente hay una tendencia en algunos neurocientíficos a alentar una concepción hegemónica de la neurociencia como guía de la educación. ${ }^{2}$ Yo me pregunto si es adecuado y deseable dejar en manos de las investigaciones neurocientíficas el modo como se debe enseñar y sobre todo la justificación de la propia práctica educativa. ¿Es la neurociencia la solución a los problemas de la práctica educativa? ¿Es la neurociencia la que debe guiar la educación?

Para intentar responder a la pregunta acerca del fin de la neuroeducación propongo introducir un nuevo elemento en la relación, la ética. ${ }^{3}$ En un primer momento puede parecer que al hacerlo se complica innecesariamente la relación, pues al haber tres elementos - educación, neurociencia y ética- en lugar de dos -educación y neurociencia- parece que las combinaciones y los puentes que se podrían establecer entre ellos son más complejos.Y esto en gran parte es verdad pues las combinaciones entre los tres enfoques se multiplican rápidamente: neuroeducación ética, neuroética educativa, neurociencia de la ética educacional, ética de la neurociencia educacional, educación de la ética de la neurociencia, educación de la neurociencia de la ética, etc. Mi hipótesis, sin embargo, es que la ética no constituye un tercero en discordia sino que realmente ella puede ayudar a esclarecer la compleja relación entre neurociencia y educación y determinar cuál es la correcta relación entre ellas en el caso específico de los fines que ha de perseguir.

2 Un ejemplo de neurocientífico que considera que la neurociencia ha de contribuir a ser guía de la práctica educativa y que es conocido en el ámbito español es el de Francisco Mora (2013a). Aunque no se le puede criticar que genere neuromitos, sin embargo, sus planteamientos, a mi juicio, adolecen del sesgo cientificista reduccionista que anula la posibilidad de la interdisciplinariedad y da a la neurociencia un papel hegemónico, aunque ello implique la negación de la libertad en la condición humana (Mora, 2013b).

3 Esta línea de investigación que introduce la ética en la relación entre neurociencias y educación ya ha sido bosquejada por algunas autoras como Adela Cortina (2011) o María José Codina (2015). 


\section{LA ÉTICA APLICADA Y LA ÉTICA FUNDAMENTAL DE LA NEUROEDUCACIÓN}

No voy a explorar todas las posibles combinaciones entre ética, educación y neurociencia porque ello excede los objetivos del presente trabajo. Sin embargo, creo que puede trazarse una importante distinción entre cuestiones de ética aplicada relacionadas con la neuroeducación y cuestiones de ética fundamental relacionadas con la neuroeducación.

En el ámbito de la neuroética es frecuente encontrar la distinción entre «ética de la neurociencia»y la «neurociencia de la ética» (Roskies, 2002: 21). Mientras que a la primera se le considera una rama de la bioética y, por lo tanto, una ética aplicada que considera las implicaciones éticas de las investigaciones neurocientíficas (Dana, 2002); la segunda, teniendo en cuenta las investigaciones y descubrimientos neurocientíficos, aborda cuestiones fundamentales de la ética tales como la relación mente-cuerpo, la libertad, la identidad, la moralidad, los métodos para acceder a las fuentes de lo moral, etc. (Cortina, 2013: 805ss.; Racine et al., 2017). ${ }^{4}$

De forma paralela y a la luz de la bibliografía mentada -aunque con ciertos matices porque la relación es más compleja-, considero que conviene diferenciar, por una parte, las cuestiones de ética aplicada a la neuroeducación que se ocupan de analizar cuáles son las implicaciones éticas de los avances en la neurociencia educacional y, por otra parte, las cuestiones que plantean cuestiones fundamentales de la educación, teniendo en cuenta los avances en neuroética.

\subsection{La ética aplicada de la neuroeducación}

Paul Howard-Jones (2010: 122-137) se refiere a una «ética neuroeducativa» para incidir en la dimensión ética de los asuntos que implican a la neurociencia con la educación y señala principalmente tres áreas. En primer lugar, el uso de medidas neurofisiológicas en la investigación educativa y el riesgo psicológico que comportan. En segundo lugar, el escrutinio y comunicación de los descubrimientos y conceptos de la neuroeducación. En tercer lugar, la responsabilidad de los responsables de políticas educativas y su obligación de tener en cuenta a los afectados.

4 Creo que son especialmente atinadas las "guías" que Racine et al. (2017) ofrecen para avanzar en la investigación acerca de la neurociencia de la ética en términos de mayor "transparencia y precisión normativa y conceptual". 
Por su parte, Hardiman et al. (2012) se refieren a «neuroética educativa» para situar el plexo de temas que flotan entre la ciencia del cerebro y la educación, remarcando que la ética en este contexto incide, por una parte, en el modo como los hallazgos científicos se transmiten a la comunidad educativa y, por otra parte, el modo como dichos hallazgos son interpretados y aplicados por los docentes en sus prácticas educativas. La neuroética educativa asume el reto de llevar adecuadamente los descubrimientos científicos al aula. Pero, para ello, hay diversos agentes que están implicados: docentes, neurocientíficos, medios de comunicación, responsables políticos e instituciones educativas. Cada uno de ellos ha de tener presente los peligros de los neuromitos que simplifican y son carne de cañón del sensacionalismo de los medios de comunicación populares. Hardiman y sus colaboradores señalan que es fundamental contar con una cuidadosa labor de traducción entre educadores y neurocientíficos.

Conviene remarcar que tanto la «ética neuroeducativa» de Howard-Jones (2010) como, sobre todo, la «neuroética educativa» de Hardinan et al. (2012) se asientan sobre el quicial de la ética aplicada. Por lo tanto, no se trata aquí de una neuroética que cuestione y analice aspectos fundamentales de la ética -neurociencia de la ética-, por ejemplo, si los educadores o educandos son libres y responsables de sus actos o no lo son. Muestra de ello es que el punto de partida tanto de Hardinan et al. (2012) como de Howard-Jones (2010) es asumir la responsabilidad por parte de los diversos agentes de trasladar adecuadamente los hallazgos neurocientíficos al aula. La neuroética educativa aquí planteada constituye una provincia de la neuroética aplicada -ética de la neurociencia-, en este caso aplicada a la práctica educativa.

\subsection{La ética fundamental de la neuroeducación}

Sin embargo, aún es posible pensar otro modo de relación más fundamental en la que la ética se vincula con la neuroeducación. Me refiero esta vez a otro tipo de cuestiones que permitan entender mejor cuál debe ser la relación interna que ha de establecerse entre educación y neurociencia. En este segundo caso, ya no se trata de reflexionar acerca de cuáles son las implicaciones éticas de determinados avances neurocientíficos o de qué forma han de ser comunicados. Antes bien, lo que surge desde este ámbito es el intento por trasladar el horizonte de reflexión y análisis logrado en la investigación en neurociencia de la ética al campo específico de la educa- 
ción. Surgen así cuestiones como la de si existe libertad o determinismo en el individuo y el modelo pedagógico que de una y otra concepción se derivan; o acerca de si la neurociencia ha de constituir la base o el fundamento de la educación; o de si la explicación evolucionista es la que ha de dar razón del fenómeno educativo; o de si todo proceso educativo se reduce a mecanismos psicológicos. Es en este segundo ámbito en el que me centro en este trabajo y más específicamente en la cuestión fundamental acerca del fin de la neuroeducación.

\section{LA CUESTIÓN FUNDAMENTAL EN TORNO AL FIN DE LA NEUROEDUCACIÓN}

Tras haber explorado los puentes entre neurociencia, educación y ética, y haber analizado la correcta relación entre ellas, llegamos al núcleo del tema que nos ocupa en este trabajo. Para ello creo que es importante recordar que toda actividad educativa ha de cuestionarse cuál es el fin que persigue, esto es, qué meta u objetivo da sentido y justifica la práctica educativa. No se trata en absoluto de una cuestión superflua o baladí sino muy necesaria y pertinente. Y lo es porque en ocasiones hay muchos otros intereses en liza como, por ejemplo, el interés económico o el de determinadas ideologías políticas que utilizan la educación para sus propios objetivos. ¿Cuál ha de ser el fin propio de la educación? Cuando hablamos de fin último de la educación, a mi juicio, no puede soslayarse que lo que justifica y le confiere su sentido último es el pleno desarrollo de la personalidad y no de cualquier manera sino fundamentándose en determinados principios y valores que regulan adecuadamente el desarrollo humano. A mi juicio, este y no otro es el fin de la educación, un fin inequívocamente ético (Gracia, 2017; Gracia et al., 2016).

La educación tendrá por objeto el pleno desarrollo de la personalidad humana y el fortalecimiento del respeto a los derechos humanos y a las libertades fundamentales; favorecerá la comprensión, la tolerancia y la amistad entre todas las naciones y todos los grupos étnicos o religiosos, y promoverá el desarrollo de las actividades de las Naciones Unidas para el mantenimiento de la paz (artículo 26, Declaración Universal de los Derechos Humanos, ONU, 1948). ${ }^{5}$

5 La Constitución Española lo expresa de forma muy similar en su artículo 27: "La educación tendrá por objeto el pleno desarrollo de la personalidad humana en el respeto a los principios democráticos de convivencia y a los derechos y libertades fundamentales". 
Cuando entra en juego la neurociencia intervienen entonces nuevos modelos explicativos del aprendizaje humano. Hay quienes defienden que la neurociencia es objetiva y neutral. No conviene perder de vista, sin embargo, que el interés de conocer el funcionamiento de los procesos cerebrales del aprendizaje es ya un tipo de interés y, a mi juicio, lo es y muy importante, pero no el único ni el rector. De hecho, la propia investigación científica presupone aspectos éticos como, por ejemplo, el compromiso ético de la comunidad científica con la verdad. Así, considerar la ciencia y la técnica como guías de las sociedades y desproveerlas de la reflexión ética acerca del horizonte ético que oriente y confiera un buen uso de dichos conocimientos científicos y técnicos es para decirlo con Habermas (1984) un modo de «ideología». ${ }^{6}$ Un buen modo de combatirla es yendo a la pregunta fundamental acerca del sentido y justificación de la actividad y los hallazgos científicos. Hay que preguntarse si a tenor de los resultados obtenidos no es desmesurado atribuir a la neurociencia capacidad normativa (Salles, 2013).

Teniendo presente el debate de la filosofía y, sobre todo, de la ética con la neurociencia y más específicamente en el ámbito de la educación (Codina, 2013; Pallarés, 2013; Conill \& Pérez Zafrilla, 2013; Conill, 2016), la pregunta que interesa replantear es, ¿debe la educación modular su objetivo e incluso supeditar sus fines al de la neurociencia? ¿Debe la neurociencia naturalizar la educación de modo que la ética resultante sea aquella que se adecúe a las pautas de conductas marcadas por la evolución? En suma, ¿cuál ha de ser el objetivo de la neuroeducación?

En su respuesta a Bowser (2016), Howard-Jones et al. (2016) afirman que «el objetivo de la neurociencia educacional es motivar al pensamiento y a la práctica educativa a partir de modelos que surgen de datos neuronales y conductuales» (Howard-Jones et al., 2016: 622). A través de estudios con neuroimagen se revelan nuevas capacidades cognitivas complejas que no eran predecibles únicamente con los datos conductuales. El ejemplo que ellos ponen está relacionado con el aprendizaje de las matemáticas y más en concreto con los números enteros positivos y negativos en niños y en adultos. Considero que este objetivo destinado a desarrollar habilidades instrumentales de cálculo - en el caso de la discalculia- o de lectura - en el caso de la dislexia- es legítimo, pero aún corto de miras si lo que nos proponemos es determinar el fin último de la educación en su conjunto.

6 Una buena crítica del naturalismo basado en la teoría moral habermasiana en el que el uso moral de la razón se identifica con el nivel posconvencional de Kohlberg puede encontrarse en Ortega (2016). 
Aclarar la dimensión ética inmanente a la actividad educativa es fundamental, sobre todo, teniendo en cuenta el auge que actualmente gozan las éticas naturalistas, ${ }^{7}$ también para explicar la educación. ¿Es la evolución la que ha de marcar la pauta y ser la norma para la educación moral? ¿Incorporar el aporte de la neurociencia en el ámbito educativo conduce a adoptar una ética evolucionista naturalista?

\section{EL SESGO NATURALISTA DE LA NEUROEDUCACIÓN MORAL DE DARCIA NARVAEZ}

La neuroeducación moral de Darcia F. Narvaez aboga por tomar como referencia de normalidad ética el comportamiento de nuestros ancestros hace treinta millones de años. El entorno de los mamíferos ancestrales ha de determinar la norma a seguir. A su juicio, el hombre occidental que ha olvidado su pasado evolutivo y no considera su naturaleza como mamífero, sufre un acuciante deterioro que tiene su origen en una pérdida de los hábitos y contextos de aprendizaje ancestrales. En la estela de la hipótesis del «cerebro triuno» de MacLean (1978), la teoría ética que defiende se basa en el trípode de la seguridad, el vínculo afectivo y la imaginación que ha sido legado al ser humano actual por la evolución. A su juicio, la importancia radica en detectar que en los primeros años de vida tiene lugar el desarrollo de estas tres dimensiones éticas, y que los contextos y el cuidado ejercido por la evolución ha de ser la pauta que seguir para un adecuado desarrollo moral desde la infancia y fundamentalmente en la infancia.

En esta línea, Narvaez elabora algunas recomendaciones sobre cómo tomar el entorno de los mamíferos humanos ancestrales como referencia y «norma» a seguir tanto en el cuidado por parte de los padres como de los profesionales de la educación. Conviene tener muy presente que esta posición busca volver al «entorno de adaptación evolutiva» (Narvaez, 2012) o «nicho de desarrollo evolucionado» (Narvaez, Hastings, Braungart-Rieker, Gettler \& Miller-Graff, 2016) y todas sus recomendaciones se basan en la

7 Entiendo por naturalismo aquel planteamiento que reduce la explicación de la conducta moral a las bases fisiológicas descubiertas por la neurociencia. Una formulación actual para definirlo podría ser: "LL]a posición que sostiene que la naturaleza —entendida como el universo físico- es todo lo que hay; en segundo lugar, que las únicas verdades básicas son las verdades de la naturaleza; y, en tercer lugar, que el único conocimiento sustantivo es el de los hechos naturales" (Audi, 2015, 28). He esbozado ya algunos argumentos en contra de diferentes versiones de ética naturalista como la de Joshua Greene (Gracia, 2016), la de Patricia Churchland (Gracia, en prensa[a]) o la de Frans De Waal. 
«asunción de que 30 millones de años de prácticas parentales son más meritorias que prácticas de padres recientemente adquiridas» (Narvaez, 2012: 153). Se trata por lo tanto de una teoría ética naturalista que entiende la «normalidad» y norma ética que ha de regular la conducta de las personas en términos de lo que la evolución ha ido legando como pauta de conducta en los mamíferos humanos. Es por lo tanto una versión de la ética del cuidado basada en los entornos de adaptación evolutiva poniendo especial énfasis en los primeros años de la infancia (Narvaez, Wang, Chen, 2016; Gleason et al., 2016).

Sin embargo, el modelo de neuroeducación moral que presenta Darcia Narvaez y sus colaboradores es a mi modo de ver deficiente. Sin duda que no se puede dejar de reconocer la importancia de los estudios en neuroeducación que nos indican qué modos de enseñanza y de aprendizaje son los más adecuados y en especial el importante papel que desempeña un entorno del cuidado para el desarrollo del carácter, sobre todo, en las primeras etapas del desarrollo humano. Sin embargo, el presupuesto naturalista de orientar la educación con vistas a los entornos de adaptación evolutivos puede ser pernicioso si lo que nos proponemos es un tipo de educación fundamentada en principios universales. De hecho, sustituir la dimensión cognitiva del juicio moral por una ética basada en la salud afectiva resulta inadecuado. Convendría recordar, ¿es suficiente y adecuada una ética basada en el «sano sentir»-desprovisto de razón? ¿No hay que argumentar y utilizar precisamente la razón para escrutar los modos de conducta y valores subyacentes para ver si realmente valen o no? La ética del cuidado naturalista entraña el problema de que toma como norma las pautas pertrechadas por la evolución y ello comporta aceptar y acoger al prójimo, pero rechazar al que no pertenece al propio grupo (Cortina, 2011).

Por otra parte, la crítica a la sociedad occidental por haber roto sus vínculos con el entorno natural y atentar contra el ecosistema y la biodiversidad es perfectamente legítima. Como también lo es la denuncia del protagonismo desmesurado y despótico que ha adquirido la racionalidad instrumental en las sociedades humanas. Ampliar la sensibilidad moral y recuperar el vínculo con la naturaleza me parece, sin lugar a duda, acertado y un desafío para las sociedades contemporáneas tan altamente tecnificadas. Pero de ahí a hacer apología de las «pequeñas comunidades de cazadores humanas» alentando el «vínculo con la vida comunal» (Narvaez, 2016: 16) va un gran trecho. No me cabe duda de que el cuidado juega un papel central en los primeros estadios del desarrollo evolutivo de la moral del niño, pero en términos éticos -y no solo biológicos o sociales- este cui- 
dado no solo se entiende en términos adaptativos. Si no ampliamos el horizonte ético al punto de vista moral en el que es posible establecer un hiato entre el valor de la naturaleza y la dignidad humana, el desarrollo moral del niño, a mi juicio, quedará lastrado. La acción - humanamentevirtuosa no atiende a factores evolutivos sino al cultivo de un carácter moral (Codina, 2015).

Desde el propio prisma evolutivo, la hipótesis del cerebro triuno que en los años setenta defendió MacLean ha sido objeto de críticas (Krubitzer y Seelke, 2012). No me voy a detener en ellas. Al hilo de la pregunta acerca del fin de la neuroeducación sí que me interesa rastrear otras formas de relación entre la educación y el enfoque evolutivo y evaluar si realmente son éticamente más adecuadas.

\section{HACIA UN NUEVO ENFOQUE EVOLUTIVO (NO NATURALISTA) DE LA EDUCACIÓN}

Tras repasar algunas de las teorías que desde Darwin se han venido dando de la evolución biológica del ser humano y de qué modo se explica el aprendizaje y la educación en el ser humano, Howard-Jones (2014) apuesta por lo que él califica como un "nuevo pensamiento» acerca de la evolución del ser humano. Se trata de un enfoque más fundamental que trata de destacar las características singulares del cerebro humano respecto al de otras especies. A su juicio, a la luz de la genética y de la arqueología es posible establecer una narrativa de la evolución en términos del cerebro y de la mente que contribuya a la comprensión de los procesos neurológicos y mentales, y que ayude a iluminar el papel de la educación para desarrollar el potencial humano como especie. Es importante destacar que Howard-Jones se aleja de la teoría de la recapitulación de planteamientos neodarwinistas que entienden el desarrollo ontogenético del cerebro en términos del desarrollo filogenético y también de la llamada psicología evolutiva. Su interés es ahondar en la perspectiva evolutiva no para desplazar la dimensión educativa moduladora de la conducta humana, sino precisamente para explorar las implicaciones que tiene este nuevo enfoque acerca de la evolución en la educación.

A mi modo de ver, este planteamiento que recoge los aportes de la perspectiva evolucionista no incurre, sin embargo, en el reduccionismo de identificar el fin y razón de ser de la ética con el fin de la evolución. Resiste a los envites del naturalismo y se cuida de no asimilar o reducir lo ético 
a los fines adaptativos de la evolución. Por mor de la claridad y atendiendo al espacio del que aquí disponemos me detendré en tres aspectos de este «nuevo enfoque» de la evolución que respeta el lugar que le corresponde a la educación ética.

En primer lugar, cabría decir que es la educación lo que hace al ser humano tan extraordinario. Somos lo que somos no solo por la evolución sino por la educación y la transmisión cultural. De hecho, lo que nos hace tan extraordinarios no es tanto la diferencia biológica legada en el organismo humano por la evolución, cuanto la trasmisión cultural que se va acumulando hasta llegar a unos niveles extraordinarios. Comparado con otras especies, lo extraordinario en el homo sapiens no es la evolución biológica sino la evolución cultural. «Es la educación la que ha transformado a un miembro de la familia de los primates, que de otro modo sería un miembro ordinario, en la especie más extraordinaria de nuestro planeta» (HowardJones, 2014: 27). En esta línea, por ejemplo, hay que explorar las bases culturales del comportamiento y las diferencias cognitivas entre razas dentro de nuestra especie humana a la luz del poderoso papel que desempeña la educación y no tanto los aspectos biológicos.

En el marco de la neuroeducación y a propósito de la relación entre biología y cultura, José Antonio Marina ${ }^{8}$ (2012) ha incidido en la relación entre biología y cultura en términos de "coevolución», pues merced a la neuroplasticidad y en virtud de la educación y su poder de transformar las estructuras del cerebro «la especie humana es capaz de dirigir su propia evolución, convierte a la educación en la gran estudiosa de ese proceso evolutivo» (Marina, 2012: 9). El aprendizaje produce cambios neuronales y la cultura cambia el cerebro, la educación irá transformando las estructuras neuronales y sus funciones. Pero el cerebro, a su vez, en todas sus singularidades individuales irá también transformando la cultura.

En segundo lugar, por tanto, conviene dejar claro que no es que se haya de adecuar la educación a patrones evolutivos como algunos enfoques evolucionistas naturalistas se empeñan en defender (Mora, 2013a: 91ss). Más bien se trata de todo lo contrario. Desde la educación, se van supliendo las deficiencias y carencias de la herencia biológica. Nuestro entorno y forma de vida ha cambiado sustancialmente en los últimos mil años mientras que nuestra base genética se mantiene sin apenas cambios. No es la evolución biológica sino la educación la que marca la pauta de qué ha de ser normal. En el caso de la dislexia y la discalculia hay argumentos para sostener que

8 Un modo de entender esta simbiogénesis cultural puede encontrarse en la epigénesis proactiva de Kathinka Evers (Evers, 2013; Pallarés-Domínguez, 2016). 
no hay una selección natural, sino que a pesar de que se produjo el cambio en el entorno y se introdujo la escritura hace varios milenios, sin embargo, sigue habiendo una elevada parte de la población que continúa teniendo dificultades (Howard-Jones, 2014: 28). ¿Cómo explicar esta inadaptación al entorno? Habría que concluir, que la normalidad o anormalidad, por lo tanto, no es algo que venga pautado por evolución biológica sino algo construido socialmente en función del entorno creado.

Un argumento similar puede hacerse en el ámbito de la ética. La normalidad ética no se ajusta a los patrones de selección natural, sino que la norma ética se superpone sobre el mecanismo evolutivo de la adaptación e incluso puede llegar a contradecirlo. En esta línea, destacados autores evolucionistas han trazado una clara distinción entre evolución biológica y evolución cultural. Elevando una crítica contra los representantes de la conocida sociobiología -J.S. Huxley, C.H.Waddington o Edward O. Wilson- por mantener una ética evolucionista reduccionista y falaz, Francisco Ayala ha sostenido que «las normas morales de acuerdo con las cuales decidimos si una acción particular es correcta o incorrecta no están especificadas por la evolución biológica sino por la evolución cultural» (Ayala, 2006: 358). Así conviene no confundir el valor biológico de la adaptación con la validez moral. El fin de la educación no ha de venir marcado por el primero sino por el segundo. La perspectiva evolutiva de la neurociencia puede ayudar a entender el origen y la evolución de determinadas predisposiciones naturales, pero de ello no se ha de seguir que lo que la naturaleza nos ha legado es éticamente bueno, ni que el valor que ha de prevalecer es el de la adaptación (Gracia, 2016). ${ }^{9}$ En el caso de la capacidad ética más que de «adaptación» cabría decir que se trata de «exaptación» (Richart, 2016).

En tercer lugar, hay que ser cauteloso y no pensar que la educación influye directamente en la evolución neurobiológica. Es cierto que el cambio de dietas o enfermedades pueden provocar cambios neurofisiológicos, pero ello solo influye de modo indirecto. La complejidad y fluidez de la cultura da a entender que más que sustratos biológicos hay capacidades humanas a través de las cuales el aprendizaje y transmisión cultural pueden propagarse (Thomas, 2013).

9 Creo que Francisco Mora (2013a: 91ss) confunde la ética con el valor adaptativo de la evolución incurriendo de este modo en el reduccionismo, hasta el punto de negar que exista la libertad en el ser humano (Mora, 2013b). ¿Realmente es posible extraer una ética universal con contenidos morales desde los códigos cerebrales? (Cortina, 2011 y 2013). 


\section{CONCLUSIÓN. NECESIDAD DE LA ÉTICA PARA ACLARAR EL FIN DE LA NEUROEDUCACIÓN}

Tras adentrarnos en el diálogo entre la neurociencia y la educación, mi conclusión es que es necesario un detenido y concienzudo análisis de la ética para aclarar cuál es el fin último que persigue la neuroeducación. Contrariamente a algunos planteamientos naturalistas que reducen la norma ética a los patrones que la evolución ha legado en nuestra biología, la reflexión neuroética no naturalista permite entender mejor el extraordinario potencial de la educación. Basándose en la neuroplasticidad y merced a la educación, el ser humano es capaz de desarrollar una ética que justifique su conducta. Esta ética ya no se fundamenta en la condición biológica heredada sino en una justificación ética de otro orden. La explicación evolucionista de la génesis del cerebro humano que aprende es muy pertinente y permite comprender el origen y singularidad de los procesos neuronales implicados en el aprendizaje. Pero esta explicación hace un flaco favor a la educación si acaba subsumiendo la ética al principio adaptacionista para la supervivencia. Porque la educación constituye un potencial extraordinario que solo se desarrolla apropiadamente con vistas al fin ético de una vida justa y plena.

\section{REFERENCIAS BIBLIOGRÁFICAS}

Ayala, Francisco (2006). La evolución de un evolucionista. Valencia: Publicaciones Universidad de Valencia.

Bowers, Jeffrey S. (2016). The practical and principled problems with educational neuroscience. Psychological Review, 123, 600-612.

Bruer,John T. (1997). Education and the brain:A bridge too far. Educational Researcher, 26, 4-16.

Codina, María José (2013). Fundamentos filosóficos de la neuroeducación. En López, Francisco Javier et al. (eds.). Bioética, neuroética, libertad y justicia (867-881). Granada: Comares.

Codina, María José (2015). Neuroeducación en virtudes cordiales. Cómo reconciliar lo que decimos con lo que hacemos. Barcelona: Octaedro. Cortina, Adela (2011). Neuroética y neuropolítica. Sugerencias para la educación moral. Madrid:Tecnos. 
Cortina, Adela (2013). Neuroética: ¿Ética fundamental o ética aplicada? En López, Francisco Javier et al. (ed.). Bioética, neuroética, libertad y justicia (802-830). Granada: Comares.

Conill, Jesús (2016). La racionalidad en perspectiva neuroética. Pensamiento, $273,767-770$.

Conill, Jesús \& Pérez, Pedro Jesús (2013). Retos actuales de la neuroética. Recerca, 13, 6-10.

Dana, Foundation (2002). Neuroetbics: Mapping The Field. Conference Proceedings, May 13-14, San Francisco, California. Nueva York: The Dana Press.

Dekker, Sanne, Lee, Nikki C., Howard-Jones, Paul \& Jolles, Jelle (2012). Neuromyths in education: Prevalence and predictors of misconceptions among teachers. Frontiers in Psychology, 3(oct.), [Article 429]. doi: 10.3389/fpsyg.2012.00429.

Goswami, Usha \& Szücs, Dénes (2011). Educational Neuroscience: Developmental mecanisms: Toward a conceptual framework. Neuroimage, 57 , 651-658.

Gracia, Javier (2016). ¿Incurre la teoría del proceso dual del juicio moral en falacia naturalista? Pensamiento, 273, 809-826.

Gracia, Javier (2017). El fundamento ético-filosófico de la educación. En Calvo, Patrici y Medina, Maria (eds.) Mirades intersubjectives en la filosofía actual (19-26). Valencia: Societat de Filosofía del País Valencià.

Gracia, Javier (en prensa). Propuesta de neuroética no naturalista. En De Tienda, Lidia et al. (Eds.). Juicio moral y democracia. Granada: Comares.

Gracia, Javier \& Gozálvez, Vicent (2016). Justificación filosófica de valores éticos y cívicos en la educación formal. Análisis crítico de la LOMCE. Teoría de la educación, 28, 1, 83-103.

Habermas, Jürgen (1984). Ciencia y técnica como ideología. Madrid:Tecnos.

Hardiman, Mariale, Rinne, Luke, Gregory, Emma \& Yarmolinska, Julia (2012). Neuroethics, Neuroeducation and Classroom teaching. Where the Brain Sciences Meet Pedagogy? Neuroethics, 5, 135-143.

Howard-Jones, Paul (2010). Introducing Neuroeducational Research. Neuroscience, education and the brain from contexts to practice. Londres: Routledge.

Howard-Jones, Paul (2014). Evolutionary Perspectives on Mind, Brain and Education. Mind, Brain and Education, vol. 8, 1, 21-33. 
Howard-Jones, Paul, Ansari, Daniel, De Smet, Bert, Laurillard, Diana, Varma, S., Butterworth, Brian, Goswami, Usa \& Thomas, Michael S. C. (2016). The Principles and Practices of Educational Neuroscience: Comment on Bowers. Psychological Review, 123, 5, 620-627.

Krubitzer, Leah A. \& Seelke, Adele M. H. (2012). Cortical evolution in mammals:The bane and beauty of phenotypic variability. En Ayala, Francisco J. (Ed.) Proceedings of the National Academy of Sciences of the United States of America (10647-10654). Irvine: University of California.

Leefmann, John, Levallois, Clement \& Hildt, Elisabeth (2016). Neuroethics 1995-2012. A Bibliometric Analysis of the Guiding Themes of an Emerging Researc Field. Frontiers in Human Neuroscience, vol. 10.

MacLean, Paul (1978). A mind of three minds: Educating the triune brain, 77th Year book of the National Society of the Study of Education. Chicago: University of Chicago Press.

Marina, José Antonio (2012). Neurociencia y educación. Participación Educativa, 1, 7-13.

Mora, Francisco (2013a). Neuroeducación. Solo se puede aprender aquello que se ama. Madrid:Tecnos.

Mora, Francisco (2013b). ¿Es la libertad una ilusión? En López, Francisco J. et al. (eds.) Bioética, Neuroética, Libertad y Justicia (831-844). Granada: Comares.

Narvaez, Darcia (2012). Moral Neuroeducation from Early Life Through the Lifespan. Neuroethics, vol. 5, n. ${ }^{\circ}$ 2. 145-157.

Narvaez, Darcia (2016). Baselines for Virtue, en Annas, J., Narvaez, D. \& Snow, N. E. (eds.). Developing the Virtues. Integrating Perspectives. Oxford: Oxford Scholarship Online.

Narvaez, Darcia, Hastings, Paul D., Braungart-Rieker, Julia M., Gettler, Lee T. \& Miller-Graff, Laura E. (2016). Young, Child Flourishing as an aim for Society. En Narvaez, Darcia, Braungart-Rieker, Julia M., Miller-Graff, Laura E., Getler, Lee T. \& Hastings, Paul D. (eds.). Contexts for Young child flourishing: Evolution, Family, and Society (347-360). Oxford: Oxford University Press.

Narvaez, Darcia, Lijuan, Wang \& Ying, Cheng (2016). The evolved developmental niche in childhood: Relation to adult psychopathology and morality. Applied Developmental Science, vol. 20, issue 4, 294-309.

ONU (1948). Declaración Universal de los Derechos Humanos, París: ONU. http://www.un.org/es/universal-declaration-human-rights/http:// www.un.org/es/universal-declaration-human-rights/ [consultado el 1 de junio 2017]. 
Ortega, César (2016). ¿Naturalizar la idea de justicia? Una respuesta crítica desde la teoría moral de Jürgen Habermas. Pensamiento, vol. 72, n. ${ }^{\circ} 273$, 827-848.

Pallarés-Domínguez, Daniel (2013). Críticas y orientaciones para el estudio de la neuroética. Recerca, 13, 85-102.

Pallarés-Domínguez, Daniel (2016). El potencial ético y educativo de la epigénesis proactiva en Kathinka Evers. Quaderns de Filosofia, vol. 3, n. ${ }^{\circ} 2,37-57$.

Racine, Eric, Dubljevic', Veljko, Jox, Ralf J., Baertschi, Bernard, Christensen, Julia F., Farisco, Michele, Jotterand, Fabrice, Kahane, Guy \& Müller, Sabine (2017). Can neuroscience contribute to practical ethics? A critical review and discussion of methodological and translational challenges of the neuroscience of ethics. Bioethics, 31, 5, 328-337.

Richart,Andrés (2016). El origen evolutivo de la agencia moral y sus implicaciones para la ética. Pensamiento, 273, 849-864.

Roskies, Adina (2002). Neuroethics for the new millennium. Neuron, 35 , 21-23.

Salles, Arleen (2013). On the normative implications of social neuroscience. Recerca, 13, 29-42. 